\title{
Sex difference in cardiac metabolism in nonischemic heart failure: Insight for prognostic value of altered cardiac metabolism
}

\author{
Hyung-Jun Im, MD, PhD, ${ }^{\mathrm{a}, \mathrm{b}}$ and $\mathrm{Gi}$ Jeong Cheon, $\mathrm{MD}, \mathrm{PhD}^{\mathrm{a}, \mathrm{c}}$ \\ ${ }^{a}$ Department of Nuclear Medicine, Seoul National University College of Medicine, Seoul, Korea \\ ${ }^{b}$ Department of Molecular Medicine and Biopharmaceutical Sciences, Graduate School of \\ Convergence Science and Technology, and College of Medicine or College of Pharmacy, Seoul \\ National University, Seoul, Korea \\ c Institute of Radiation Medicine, Seoul National University College of Medicine, Seoul, Korea
}

Received Mar 25, 2016; accepted Mar 25, 2016

doi: 10.1007/s12350-016-0489-0

\section{See related article, pp. 1226-1235}

Heart has the highest metabolic demands than any other organs. To maintain contractile function of the heart, constant and rapid production of adenosine triphosphate (ATP) is needed. Fatty acid (FA) and glucose are the main fuels for production of the ATPs by mitochondrial oxidative phosphorylation. In normal condition, $60 \%$ to $90 \%$ of total ATP is made from FA and the other from glucose or lactate. In a failing heart, cardiac metabolism is profoundly altered, and majority of the studies suggest that FA metabolism decreases. However, it is not clear and still in controversy whether the alteration in cardiac metabolism in a failing heart is an adaptation or maladaptation. ${ }^{1}{ }^{3}$

In this issue of the Journal, Kadkhodayan et al compared myocardial blood flow (MBF), FA metabolism, and glucose metabolism between men and women with nonischemic $\mathrm{HF}$, and found that MBF and FA metabolism were higher in women than men, which were independent of age, obesity, and insulin resistance. Although higher MBF and FA metabolism in women are well known, ${ }^{4}$ this study firstly showed that the sex difference of MBF and FA metabolism still stands true in subjects with nonischemic HF. Also the authors found

Reprint requests: Gi Jeong Cheon, MD, PhD, Department of Nuclear Medicine, Seoul National University College of Medicine, 101 Daehak-ro, Jongno-gu, Seoul, 03080, Korea; larrycheon@ gmail.com

J Nucl Cardiol 2017;24:1236-8.

$1071-3581 / \$ 34.00$

Copyright (c) 2016 American Society of Nuclear Cardiology. that high MBF was associated with the prognosis. Although, FA metabolism did not show significant association with survival in this study, there has been a hypothesis that decreased FA metabolism in nonischemic $\mathrm{HF}$ is a maladaptation. In particular, recent mouse studies indicate that lower FA metabolism can exacerbate nonischemic heart failure. ${ }^{5,6}$ Also, 'obesity paradox' is supporting the hypothesis, which is the observation that obese heart failure patients have better prognosis than nonobese heart failure patients even though obesity is risk factor for heart failure. ${ }^{7}$ In this study, it is hard to conclude the association between high FA metabolism and prognosis, because the size of this study was too small and potential confounding factors including perfusion reserve were not evaluated. Thus, the hypothesis on association between FA metabolism and prognosis in nonischemic HF should be confirmed in a larger study with adjustment of other prognostic markers especially sex and perfusion reserve.

In young and healthy subjects, women have lower glucose extraction fraction and utilization than men, ${ }^{8}$ in contrast, sex difference of glucose metabolism was not found in patients with nonischemic HF in this study. This result could reinforce the previous finding that FA and glucose metabolism are not always counterbalanced in HF. ${ }^{6,9}$ Previous studies on glucose metabolism in HF are less consistent, ${ }^{1}$ and the consequence of shifting toward high glucose metabolism in HF is also in controversy. ${ }^{3}$ In several studies using mice, increased glucose utilization showed favorable cardiac outcome. ${ }^{10,11}$ In contrast, one recent prospective clinical study showed that high FDG uptake in right ventricle predicted worse outcome in patients with pulmonary arterial hypertension. Meanwhile, FDG PET/CT or MR can also be utilized to assess inflammatory activity in 
Table 1. Radiotracers for evaluation of FA metabolism

\begin{tabular}{llll}
\hline Radionuclide & Half-life & Compound & \multicolumn{1}{c}{ Use } \\
\hline $\begin{array}{l}\text { SPECT } \\
{ }^{123} \mathrm{I}\end{array}$ & 13.3 hours & IPPA & FA uptake, oxidation, and storage \\
& & BMIPP & FA storage \\
PET & 20.4 minutes & Palmitate & FA uptake, oxidation, and storage \\
${ }^{11} \mathrm{C}$ & 110 minutes & FTHA & FA uptake and oxidation \\
${ }^{18} \mathrm{~F}$ & FTP & FA uptake and oxidation \\
& & FCPHA & FA uptake and oxidation
\end{tabular}

pericardial fat or visceral fat which could be associated with the risk of heart diseases. ${ }^{12}-{ }^{15}$ Thus, prognostic value of the integrated information from FDG PET/CT or PET/MR is worth to be tested in future studies.

Multiple tracers have been developed to assess cardiac FA metabolism (Table 1). FAs enter to myocardium by either passive diffusion or by proteinmediated transport, and activated to fatty acyl-coenzyme A (CoA) by fatty acyl-CoA synthase (FACS) in cytoplasm. Fatty acyl-CoA is either stored to triglyceride or converted to long-chain acylcarnitine. Long-chain acylcarnitine is transported to mitochondria, and processed by beta oxidation. Radiolabeled single-branch FAs such as ${ }^{123}$ I-15-(p-iodophenyl)-pentadecanoic acid (IPPA) or ${ }^{11} \mathrm{C}$-palmitate follows the steps of normal FA metabolism, thus we can evaluate FA uptake, storage, and beta oxidation with the tracers. The branched FAs such as ${ }^{123}$ I-beta-methyl-p-iodophenylpentadecanoic acid (BMIPP) can only be used for evaluation of FA storage, since alkyl branching of the tracers inhibits beta oxidation. Several modified FA tracers with ${ }^{18} \mathrm{~F}$ labeling such as 14(R,S)-[18F]-fluoro-6-thiaheptadecanoic acid (FTHA), ${ }^{16}$ 16- ${ }^{18}$ F-fluoro-4-thia-palmitate (FTP), ${ }^{17}$ Trans9(RS)- ${ }^{18}$ F-fluoro-3,4(RS,RS) methyleneheptadecanoic acid $(\mathrm{FCPHA})^{18}$ are trapped during beta oxidation, therefore can be used in evaluation of FA uptake and beta oxidation partially. ${ }^{11} \mathrm{C}$ labeled palmitate $\left({ }^{11} \mathrm{C}\right.$-palmitate $)$ is the most commonly used tracer for FA metabolism assessment and mathematical modeling to assess FA uptake, oxidation, and storage using ${ }^{11} \mathrm{C}$-palmitate are well established. ${ }^{19}$ The main advantages of ${ }^{11} \mathrm{C}$-palmitate is that the kinetics of the tracer is the same with the unlabeled palmitate which is a long-chain saturated FA. On the other hand, rapid clearance and short half-life of ${ }^{11} \mathrm{C}$-palmitate results in relatively poor image quality. Also, kinetic analysis is easier in tracers which are stored or trapped in myocardium than ones undergo beta oxidation and wash out. Accordingly, above-mentioned ${ }^{18} \mathrm{~F}$ labeled tracers have advantages over ${ }^{11} \mathrm{C}$-palmitate regarding ease of kinetic modeling, longer physical half- life, and better image quality. Selection of appropriate tracer would be of importance for prospective trial for evaluation of prognostic value of cardiac metabolism and therapeutic trials. For example, for an initial exploratory study, a tracer with wide range of applications such as ${ }^{11} \mathrm{C}$-Palmitate could be the tracer of choice, while ${ }^{18} \mathrm{~F}$ labeled tracers will be better choices for a study to evaluate difference in FA uptake and beta oxidation, for ease of analysis and better image quality.

Modulation of cardiac metabolism could be an early treatment target for failing heart and already has been attempted in several clinical trials. However, controversies remained whether increasing FA, decreasing FA, increasing glucose, or decreasing glucose metabolism is more beneficial to prevent progression of HF. Prior to test efficacy of drugs which alter cardiac metabolism in $\mathrm{HF}$, larger case study should be done to validate the association between prognosis and cardiac FA/glucose metabolism. Also, appropriate FA metabolism tracer should be carefully selected for the future clinical trials.

\section{Reference}

1. Doenst T, Nguyen TD, Abel ED. Cardiac metabolism in heart failure: Implications beyond ATP production. Circ Res 2013;113:709-24

2. Stanley WC, Recchia FA, Lopaschuk GD. Myocardial substrate metabolism in the normal and failing heart. Physiol Rev 2005;85:1093-129.

3. Pascual F, Coleman RA. Fuel availability and fate in cardiac metabolism: A tale of two substrates. Biochim Biophys Acta Mol Cell Biol Lipids 2016;. doi:10.1016/j.bbalip.2016.03.014.

4. Duvernoy CS, Meyer C, Seifert-Klauss V, Dayanikli F, Matsunari I, Rattenhuber J, et al. Gender differences in myocardial blood flow dynamics: Lipid profile and hemodynamic effects. J Am Coll Cardiol 1999;33:463-70.

5. Paul DS, Grevengoed TJ, Pascual F, Ellis JM, Willis MS, Coleman RA. Deficiency of cardiac Acyl-CoA synthetase-1 induces diastolic dysfunction, but pathologic hypertrophy is reversed by rapamycin. Biochim Biophys Acta 2014;1841:880-7.

6. Abdurrachim D, Luiken JJ, Nicolay K, Glatz JF, Prompers JJ, Nabben M. Good and bad consequences of altered fatty acid 
metabolism in heart failure: Evidence from mouse models. Cardiovasc Res 2015;106:194-205.

7. Lavie CJ, Alpert MA, Arena R, Mehra MR, Milani RV, Ventura HO. Impact of obesity and the obesity paradox on prevalence and prognosis in heart failure. JACC Heart Fail 2013;1:93-102.

8. Peterson LR, Soto PF, Herrero P, Schechtman KB, Dence C, Gropler RJ. Sex differences in myocardial oxygen and glucose metabolism. J Nucl Cardiol 2007;14:573-81.

9. Peterson LR, Soto PF, Herrero P, Mohammed BS, Avidan MS, Schechtman $\mathrm{KB}$, et al. Impact of gender on the myocardial metabolic response to obesity. JACC Cardiovasc Imaging 2008;1:424-33.

10. Luptak I, Yan J, Cui L, Jain M, Liao R, Tian R. Long-term effects of increased glucose entry on mouse hearts during normal aging and ischemic stress. Circulation 2007;116:901-9.

11. Luptak I, Balschi JA, Xing Y, Leone TC, Kelly DP, Tian R. Decreased contractile and metabolic reserve in peroxisome proliferator-activated receptor-alpha-null hearts can be rescued by increasing glucose transport and utilization. Circulation 2005; 112:2339-46.

12. Im HJ, Paeng JC, Cheon GJ, Kim EE, Lee JS, Goo JM, et al. Feasibility of simultaneous 18F-FDG PET/MRI for the quantitative volumetric and metabolic measurements of abdominal fat tissues using fat segmentation. Nucl Med Commun 2016.

13. Uribarri J, Cai W, Woodward M, Tripp E, Goldberg L, Pyzik R, et al. Elevated serum advanced glycation endproducts in obese indicate risk for the metabolic syndrome: A link between healthy and unhealthy obesity? J Clin Endocrinol Metab 2015;100:195766.

14. Iacobellis G, Zaki MC, Garcia D, Willens HJ. Epicardial fat in atrial fibrillation and heart failure. Horm Metab Res 2014;46:58790.

15. Iacobellis G. Local and systemic effects of the multifaceted epicardial adipose tissue depot. Nat Rev Endocrinol 2015;11:363-71.

16. Schulz G, von Dahl J, Kaiser HJ, Koch KC, Sabri O, Banneitz L, et al. Imaging of beta-oxidation by static PET with 14(R,S)-[18F]fluoro-6-thiaheptadecanoic acid (FTHA) in patients with advanced coronary heart disease: A comparison with 18FDG-PET and 99Tcm-MIBI SPET. Nucl Med Commun 1996;17:1057-64.

17. DeGrado TR, Kitapci MT, Wang S, Ying J, Lopaschuk GD. Validation of 18F-fluoro-4-thia-palmitate as a PET probe for myocardial fatty acid oxidation: Effects of hypoxia and composition of exogenous fatty acids. J Nucl Med 2006;47:173-81.

18. Shoup TM, Elmaleh DR, Bonab AA, Fischman AJ. Evaluation of trans-9-18F-fluoro-3,4-methyleneheptadecanoic acid as a PET tracer for myocardial fatty acid imaging. J Nucl Med 2005;46:297304.

19. Bergmann SR, Weinheimer CJ, Markham J, Herrero P. Quantitation of myocardial fatty acid metabolism using PET. J Nucl Med 1996;37:1723-30 